\title{
Involvement of calcitonin gene-related peptide in the modulation of human myometrial contractility during pregnancy
}

\author{
Yuan-Lin Dong, ${ }^{1}$ Li Fang, ${ }^{1}$ Sudhir Kondapaka, ${ }^{1}$ Pandu R. Gangula, ${ }^{1}$ \\ Sunil J. Wimalawansa, ${ }^{2}$ and Chandrasekhar Yallampalli ${ }^{1}$ \\ ${ }^{1}$ Department of Obstetrics and Gynecology, and \\ ${ }^{2}$ Department of Internal Medicine, University of Texas Medical Branch, Galveston, Texas 77555, USA \\ Address correspondence to: Chandrasekhar Yallampalli, Department of Obstetrics and Gynecology, \\ University of Texas Medical Branch, 301 University Boulevard, Route 1062, Galveston, Texas 77555-1062, USA. \\ Phone: (409) 772-7593; Fax: (409) 747-0475; E-mail: chyallam@utmb.edu.
}

Received for publication January 20, 1999, and accepted in revised form July 29, 1999.

Calcitonin gene-related peptide (CGRP) is a potent vasodilator and relaxes smooth muscle of a variety of tissues, but the effects of CGRP on human myometrial contractions and the changes in CGRP receptors (CGRP-Rs) in human myometrium have not been described. We report that CGRP induced dose-dependent relaxation in spontaneously contracting myometrium from pregnant women. This relaxation effect is diminished in myometrium obtained from patients during labor and in the nonpregnant state. CGRP-induced relaxations are inhibited by a CGRP-R antagonist (CGRP $\left.{ }_{8-37}\right)$, a soluble guanylate cyclase inhibitor $\left(\mathrm{LY}_{83583}\right)$, and a nitric oxide synthase inhibitor (L-NAME). Both Western blotting and mRNA analysis showed that CGRP-Rs are present in human myometrium, and that the expression of these receptors is increased during pregnancy and decreased during term labor. Immunofluorescent staining revealed that CGRP-Rs are abundant in the myometrial cells of pregnant women who are not in labor, and are minimal in uterine specimens from women in labor and in the nonpregnant state. We conclude that increased CGRP-Rs in myometrium, and resulting enhanced myometrial sensitivity to CGRP, may play a role in maintaining human myometrium in a quiescent state during pregnancy, and that a decline in the CGRP-Rs at term could contribute to the initiation of labor.

J. Clin. Invest. 104:559-565 (1999).

\section{Introduction}

Uterine quiescence is a requirement for the successful completion of term gestation. Failure to maintain uterine relaxation often results in preterm delivery one of the leading causes of infant mortality and morbidity. Studies have shown that the quiescent state of the myometrium is maintained not only by absence or low levels of stimulatory factors, such as oxytocin receptors (1), gap junction channels (2), and $\alpha$-adrenergic receptors (3), but also by an enhancement of inhibitory factors, including nitric oxide (4). However, little research has been done to define the mechanisms that maintain myometrial relaxation during pregnan$c y$, compared with research on those mechanisms that promote the onset of labor.

Calcitonin gene-related peptide (CGRP) is a 37residue neuropeptide that results from tissue-specific alternative splicing of the primary RNA transcript of the calcitonin/CGRP gene (5). The vasodilator effects of CGRP have been demonstrated in the human fetalplacental circulation (6). With immunocytochemical techniques, CGRP-immunoreactive nerve fibers were found in rat and human uterus $(7,8)$; CGRP relaxes contractile activity of the uterus in rats and humans $(7$, 8). Therefore, CGRP is proposed to be a modulator of myometrial contractility during gestation. This study investigated whether uterine relaxation responses to CGRP are differentially regulated in humans during pregnancy and labor, and if the actions of CGRP are mediated by changes in CGRP receptors (CGRP-Rs).

\section{Methods}

Human subjects. The medical procedures and the process of consent for this study were approved by the Institutional Review Board of the University of Texas Medical Branch (UTMB). All human subjects, who were patients admitted to UTMB, were either women undergoing cesarean sections or nonpregnant women having hysterectomies. All provided written informed consent. Information on each subject regarding dates of pregnancy, number of pregnancies, age, prior drug treatment, and uterine contractions was collected and stored in a computerized database for later review. Specimens from each subject were taken from the lower uterine segment at the site of incision through the uterine wall. A specimen of approximately $2.0 \times 0.6 \times 0.6 \mathrm{~cm}$ was collected and divided into smaller portions $(1.0 \times$ $0.2 \times 0.2 \mathrm{~cm})$. Once each tissue sample was collected, it was assigned a number to blind the sample and to ensure confidentiality. 

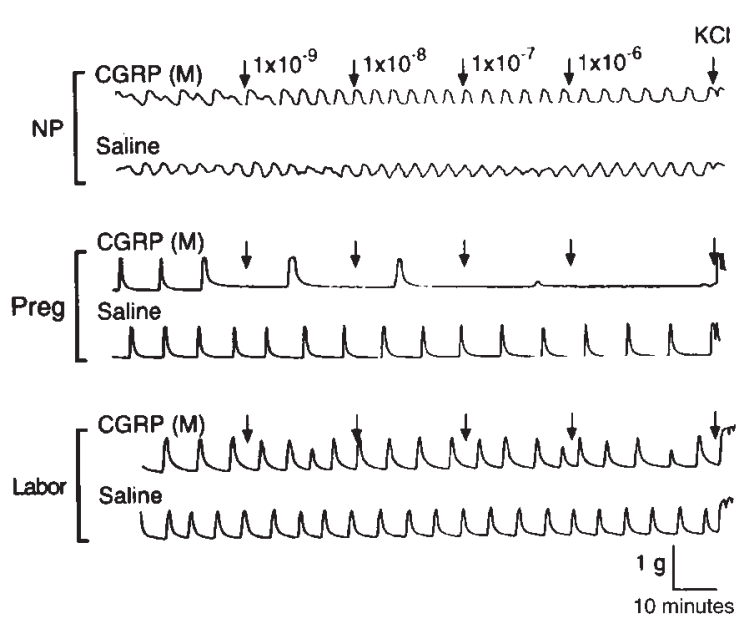

Figure 1

Relaxation response to CGRP of spontaneously contracting myometrium from women in the nonpregnant state (NP), during pregnancy but not in labor (Preg), and in labor (Labor). CGRP was added to muscle baths at increasing concentrations from $10^{-9}$ to $10^{-6}$ M. Representative tracings of contractile activity from each group (6 patients each) are presented. $\mathrm{KCl}, 45 \mathrm{mmol} / \mathrm{L}$.

In vitro contractility measurements. Relaxation responses to CGRP of human myometrium from women during pregnancy, in spontaneous term labor, or in the nonpregnant state were assessed by the procedures described previously (9). Myometrial strips measuring $1.0 \times 0.2 \times 0.2 \mathrm{~cm}$ were dissected, rinsed, and placed in $10-\mathrm{mL}$ muscle baths containing Krebs solution, which consisted of (in mmol/L) $116 \mathrm{NaCl}, 5.4 \mathrm{KCl}, 2.5 \mathrm{CaCl}_{2}$, $12 \mathrm{NaH}_{2} \mathrm{PO}_{4}, 11.2$ D-glucose, and $22 \mathrm{NaHCO}_{3}(\mathrm{pH} 7.4$ ). This solution was maintained at $37^{\circ} \mathrm{C}$ and bubbled continuously with $95 \% \mathrm{O}_{2}$ and $5 \% \mathrm{CO}_{2}$. A resting force of $1.25 \mathrm{~g}$ was placed on each strip, and the tissues were allowed to equilibrate for 1 hour before application of agents. Mean force developed per unit time by the strips was determined by measuring the area under the contraction curves during the period of CGRP administration, using a computerized MacLab system (AD Instruments, Castle Hill, Australia). The percent change in area caused by each dose of CGRP (concentration range: $10^{-9}$ to $10^{-6} \mathrm{M}$ ) was determined, and the mean and SEM of data from 6 patients were calculated. These data were compared with the same measurements taken in the presence of CGRP antagonist $\left(\mathrm{CGRP}_{8-37}\right)$, soluble guanylate cyclase inhibitor $\left(\mathrm{LY}_{83583}\right)$, or nitric oxide synthase inhibitor $\left(N^{\mathrm{G}}\right.$-nitro-L-arginine methyl ester [LNAME]). We then examined the relaxation effects of CGRP in oxytocin-induced uterine contractions, and calculated $\mathrm{IC}_{50}$ of CGRP for oxytocin-induced myometrial contractions. We also assessed $\mathrm{IC}_{50}$ values for $\mathrm{CGRP}_{8-37}, \mathrm{LY}_{83583}$, and L-NAME to block the myometrial relaxations induced by CGRP $\left(10^{-6} \mathrm{M}\right)$. All studies for determination of $\mathrm{IC}_{50}$ values were carried out using myometrial tissues from pregnant women not in labor, a stage with maximal relaxation of the myometrium. Human CGRP and CGRP 8-37 $_{\text {were synthesized by means }}$ of solid-phase chemistry by one of the authors (S.J. Wimalawansa), and cyclized, purified, and fully characterized by amino acid analysis sequencing and fast atom bombardment mass spectrophotometry (5).

Western blotting. SDS-PAGE was carried out on a $7.5 \%$ Tris-HCl Ready Gel using a Mini-PROTEAN II gel apparatus (Bio-Rad Laboratories Inc., Richmond, California, USA) (10). Ten microliters of lysate from human myometrial tissues containing $40 \mu \mathrm{g}$ of protein was loaded alongside broad-range prestained SDS-PAGE standards and electrophoresed. After electrophoretic transfer at $100 \mathrm{~V}$ for 70 minutes, the PVDF membrane (Bio-Rad Laboratories Inc.) was stained with $0.5 \%$ amido black for 5-10 minutes, followed by destaining until the bands stained for proteins were clearly distinguishable from the background. The PVDF membrane was then blocked with $5 \%$ nonfat dry milk, and incubated with primary $\mathrm{mAb}$ against CGRP-Rs at a dilution of 1:1,000 for 1 hour at room temperature. After washing, the blots were incubated with horseradish peroxidase-conjugated anti-mouse IgG for 1 hour at room temperature. The blots were then washed and subjected to enhanced chemiluminescence using the Western blotting detection system from Amersham Life Sciences Inc. (Arlington Heights, Illinois, USA). Autoradiograph film was applied to the blots until satisfactory exposure was achieved. The bands with the predicted size of 60 $\mathrm{kDa}$ were densitometrically scanned and analyzed using a SigmaGel analysis system (SPSS Science, Chicago, Illi-

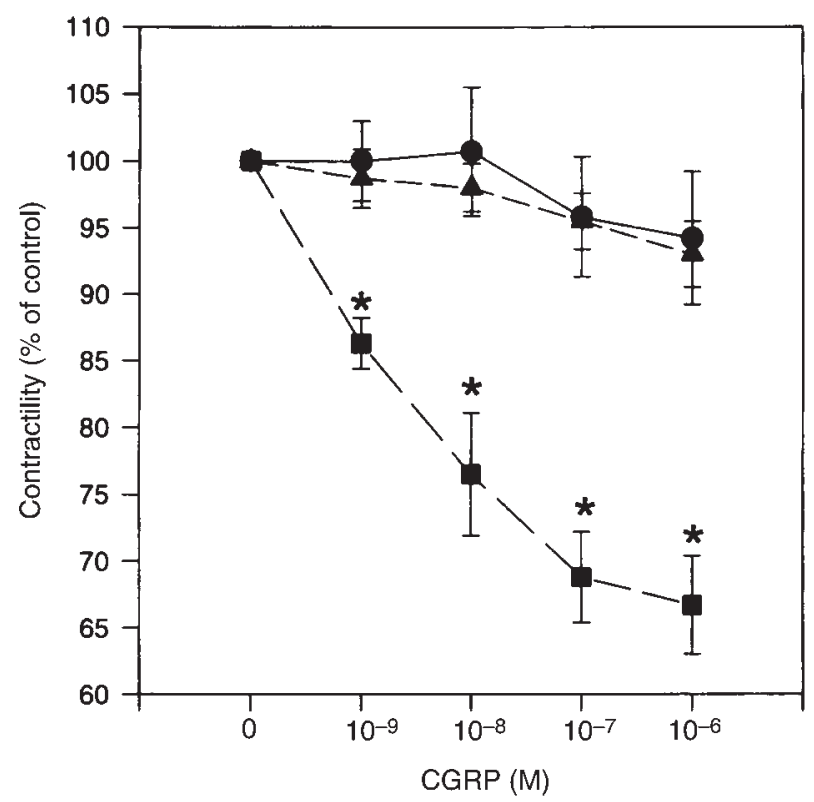

Figure 2

CGRP relaxation dose-response curves for spontaneously contracting myometrium from women in the nonpregnant state (circles), during pregnancy but not in labor (squares), and in labor (triangles). Relaxation responses, expressed as percent of control activity at each dose of CGRP applied, were analyzed by repeated-measures ANOVA among 3 groups. Each point represents mean \pm SEM for 6 patients. Asterisks indicate a statistically significant difference vs. other 2 groups ( $P<0.05$ by ANOVA followed by Bonferroni $t$ test). 


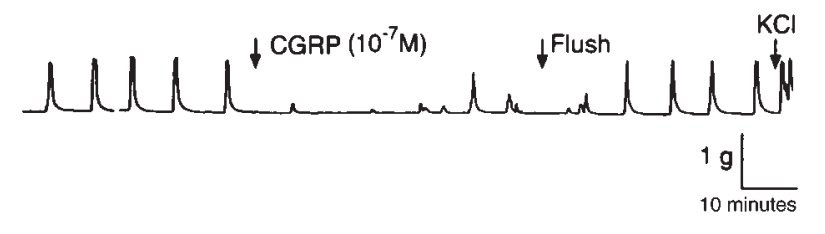

Figure 3

Representative tracing of the effect of CGRP on spontaneous myometrial contractions. This myometrial strip is from a pregnant woman not in labor. $\mathrm{KCl}, 45 \mathrm{mmol} / \mathrm{L}$.

nois, USA). Mouse mAb's against the CGRP-R were prepared from purified CGRP-Rs from cerebellum, and characterized as described previously $(11,12)$. These antibodies have been screened by ELISA, Western blot analysis, and immunohistochemistry (13).

$R T-P C R$. Total cellular RNA was extracted from the human myometrium by a single-step guanidine thiocyanate method (14) using Trizol reagent (GIBCO BRL, Gaithersburg, Maryland, USA). First-strand cDNA synthesis was primed with oligo-dT (using $2 \mu \mathrm{g}$ of total extranuclear RNA with $10 \mathrm{U}$ of reverse transcriptase and oligo-dT 12-18 [GIBCO BRL] as primer) at $42^{\circ} \mathrm{C}$ for 40 minutes, as described previously (15). Ten percent of the resulting cDNA was used for amplification by PCR with 35 cycles. PCR primers were derived from the published sequences of human CGRP-1 receptor (16). The primers used for amplification of the housekeeping gene, $\beta$-actin, were derived from the rat $\beta$-actin cDNA sequence (17). The specific primers used for amplification were as follows. Human CGRP-1 receptor: forward primer 5'-TCTGGTTCTCTTCCCTTTTTTTATG-3', reverse primer 5'-GTCCATGTTCTGTTGCTTGCTG-3' (with an expected amplified length of $380 \mathrm{bp}$ ); and $\beta$-actin: forward primer 5'-GTCGACAACGGCTCCGGCA-3', reverse primer 5'-GTCAGGTCCCGGCCAGCCA-3' (with an expected length of $530 \mathrm{bp}$ ).

PCR products, with the use of these primers, were identical to those of published sequences of human CGRP-1 receptor (16), and were confirmed by direct double-strand sequencing (fmol DNA Sequencing System; Promega Corp., Madison, Wisconsin, USA) (data not shown). The relative concentrations of CGRP-R mRNA were determined by densitometric analysis of the ethidium bromide-stained reaction products, using the SigmaGel analysis system. The results are expressed as the ratio of the densitometric readings for CGRP-R mRNA to $\beta$-actin mRNA readings; therefore, the results indicate relative changes in myometrial CGRP-R mRNA levels.

Immunofluorescent localization of CGRP-R. Immunofluorescent staining was performed by a modified immunofluorescence protocol (4). Five-micrometer paraffinembedded sections from the myometrium were cut and fixed. Five percent normal horse serum and avidin/biotin blocking buffer were applied to slides to reduce nonspecific binding. Primary (CGRP-R) $\mathrm{mAb}$ in PBS buffer was added to slides, incubated for 90 minutes at $22^{\circ} \mathrm{C}$, and
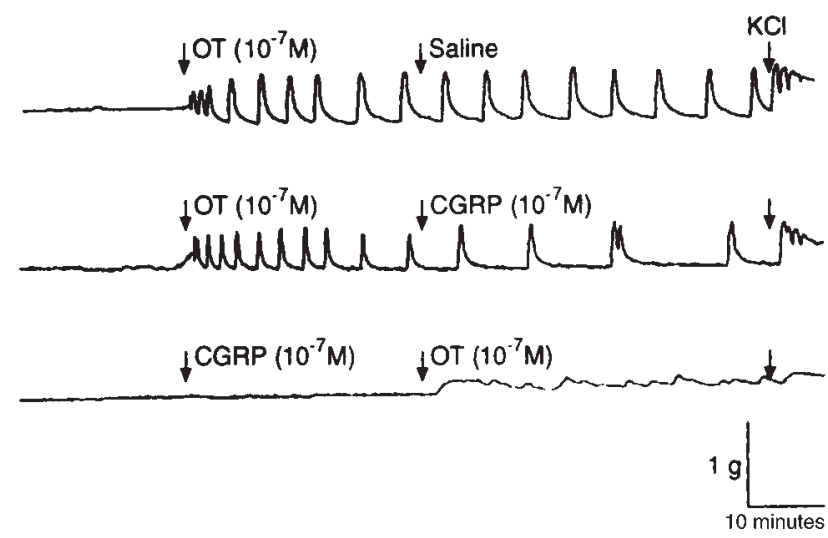

\section{Figure 4}

Representative tracing of the effect of CGRP on myometrial contractility induced by oxytocin (OT). This myometrial strip is from a pregnant woman not in labor. $\mathrm{KCl}, 45 \mathrm{mmol} / \mathrm{L}$.

then washed in PBS. Slides were then incubated with biotinylated horse anti-mouse IgG (Vector Laboratories, Burlingame, California, USA) for 45 minutes at $22^{\circ} \mathrm{C}$. After the slides were washed in PBS, the detection steps were performed by incubation of slides with Fluorescein Avidin D (Vector Laboratories) for 1 hour at $22^{\circ} \mathrm{C}$. Slides were then washed at least 4 times in PBS buffer, and propidium iodide (Boehringer Mannheim Biochemicals Inc., Indianapolis, Indiana, USA) in PBS was applied to the slides as counterstain for viewing cellular nuclei. After washing in PBS buffer, slides were mounted with VECTASHIELD mounting medium (Vector Laboratories) and viewed under a Nikon fluorescent microscope (Nikon Corp., Dallas, Texas, USA).

Statistics. Results are expressed as mean \pm SEM. Data were analyzed for statistical differences using the Student's $t$ test or ANOVA followed by Bonferroni $t$ test. When necessary, the dose-response curves for the areas under contraction curves were plotted against the log of the agent dose, and then contraction activity $\mathrm{IC}_{50}$ was calculated. These were found to be normally distributed. We then analyzed the data by ANOVA. Differences were considered significant at $P<0.05$.

\section{Results}

CGRP inhibited spontaneous contractions of human myometrium during pregnancy in a dose-dependent fashion. Application of CGRP (ranging from 10-9 to 10-6 M) to muscle strips caused relaxation of the spontaneous contractile activi-

Table 1

$\mathrm{IC}_{50}$ in human myometrium from pregnant women not in labor ${ }^{\mathrm{A}}$

\begin{tabular}{lc}
\hline Group & $\mathrm{IC}_{50}$ \\
CGRP in oxytocin-induced contraction & $5.3 \pm 0.1$ \\
CGRP $_{8-37}$ in CGRP relaxant action & $6.1 \pm 0.3$ \\
LY $_{83583}$ in CGRP relaxant action & $6.5 \pm 0.3$ \\
L-NAME in CGRP relaxant action & $3.5 \pm 0.3$
\end{tabular}

Values are mean \pm SEM. ${ }^{A}$ Expressed as negative log concentration in moles per liter. 

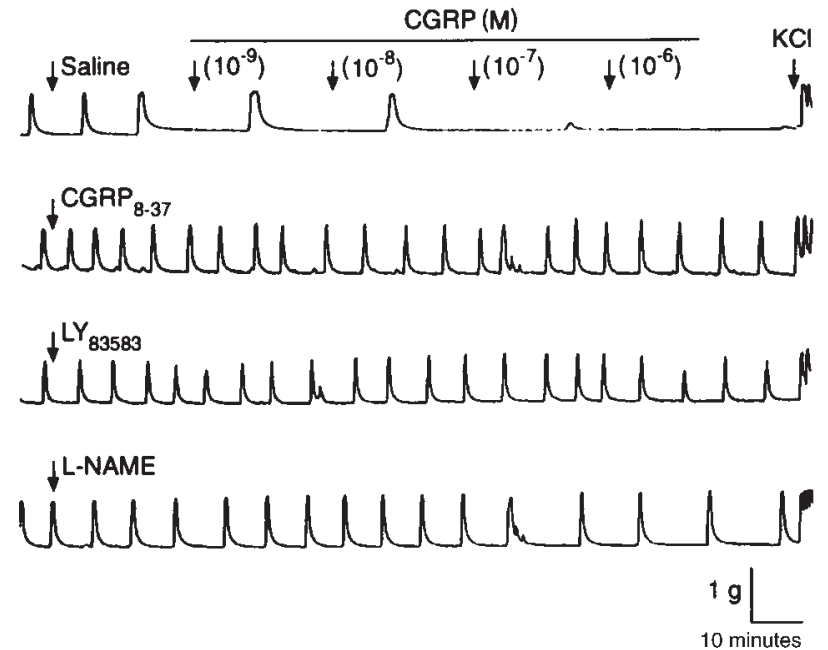

Figure 5

Relaxation responses to CGRP of spontaneously contracting myometrium from pregnant women not in labor. CGRP was added to the muscle bath at increasing concentrations from $10^{-9}$ to $10^{-6} \mathrm{M}$ in the presence of saline, $\mathrm{CGRP}_{8-37}\left(10^{-5} \mathrm{M}\right), \mathrm{LY}_{83583}\left(10^{-5} \mathrm{M}\right)$, and $\mathrm{L}-$ NAME $\left(10^{-4} \mathrm{M}\right)$. Representative tracings of contractile activity from each group ( 6 patients each) are presented. $\mathrm{KCl}, 45 \mathrm{mmol} / \mathrm{L}$.

ty of myometrium obtained from women during pregnancy (39.1 \pm 0.2 weeks; Figures 1 and 2$)$. The inhibitory effect developed rapidly and was easily washed out (Figure 3). However, the relaxation responses of the myometrium to CGRP were substantially reduced for tissues obtained from patients during labor and in the nonpregnant state $(\mathrm{P}<0.01$; Figures 1 and 2$)$, suggesting that the sensitivity of the myometrium to CGRP is increased during pregnancy and decreased during labor. CGRP inhibited oxytocin-induced contractions of the buman myometrium during pregnancy. CGRP demonstrated a significant relaxant effect in myometrium that was preconstricted with oxytocin $\left(10^{-7} \mathrm{M}\right)$. A representative data set is illustrated in Figure 4. Addition of CGRP $\left(10^{-7} \mathrm{M}\right)$ to the muscle bath, even before oxytocin application, significantly depressed oxytocin-induced contractile activity as well (Figure 4). Furthermore, CGRP inhibited the oxytocin-induced myometrial contractility in a dose-dependent manner (Table 1).

$C_{\text {CGRP }}{ }_{837}, L Y_{83583}$, and L-NAME inbibited CGRP-induced myometrial relaxation. The relaxation effects of CGRP on myometrial strips were profoundly inhibited $(P<0.01)$ by $\mathrm{CGRP}_{8-37}$, a specific antagonist of the CGRP-1 receptor, indicating the involvement of the CGRP-1 receptor in the action of CGRP on the human myometrium. Similarly, CGRP-induced relaxations are inhibited by a soluble guanylate cyclase inhibitor $\left(\mathrm{LY}_{83583}\right.$; Figures 5 and 6 , Table 1), suggesting that the cGMP pathway is involved in CGRP-induced relaxation of human uterine tissue. The effects of CGRP $\mathrm{C}_{8-37}$ and $\mathrm{LY}_{83583}$ were dose-dependent; the $\mathrm{IC}_{50}$ values were calculated and are presented in Table 1. Exposure of the myometrial strips to L-NAME, an inhibitor of nitric oxide synthase, at a concentration of $10^{-4} \mathrm{M}$ did not completely inhibit the relaxant effects of CGRP (Figures 5 and 6). However, CGRP-induced relaxations were inhibited by $50 \%$ when higher concentrations of L-NAME $(3.5 \pm 0.3 \mathrm{~mol} / \mathrm{L}$, negative log concentration) were used, indicating the involvement of nitric oxide in CGRP-induced relaxation of human myometrium (Table 1).

The expression of CGRP-R protein and CGRP-R mRNA in myometrium is increased during pregnancy and decreased during labor at term. To determine whether pregnancy-related relaxation of human myometrium by CGRP is due to changes in CGRP-R levels, we measured the levels of CGRP-R protein and mRNA in human myometrium by Western blot and RTPCR. As shown in Figure 7, a single band of CGRP-R was obtained, with the predicted size of $60 \mathrm{kDa}$. The amido-black staining shows a band of myosin at 202 $\mathrm{kDa}$. No significant changes were observed in this protein band between various experimental groups, indicating a similar amount of muscle protein in each group. In contrast, the density of CGRP-R protein was significantly higher in myometrium from pregnant women who were not in labor $(37.3 \pm 8.0$ densitometric units) compared with nonpregnant patients $(19.0 \pm 2.6$; $P<0.01)$, and profoundly declined during labor $(17.3 \pm$ 2.4; $P<0.01$ ). As shown in Figure 8, a single band for CGRP-R mRNA was obtained, with the predicted size of $380 \mathrm{bp}$. The relative changes in CGRP-R mRNA in the myometrium, expressed as a ratio of CGRP-R mRNA to $\beta$-actin mRNA, showed a significant increase in preg-

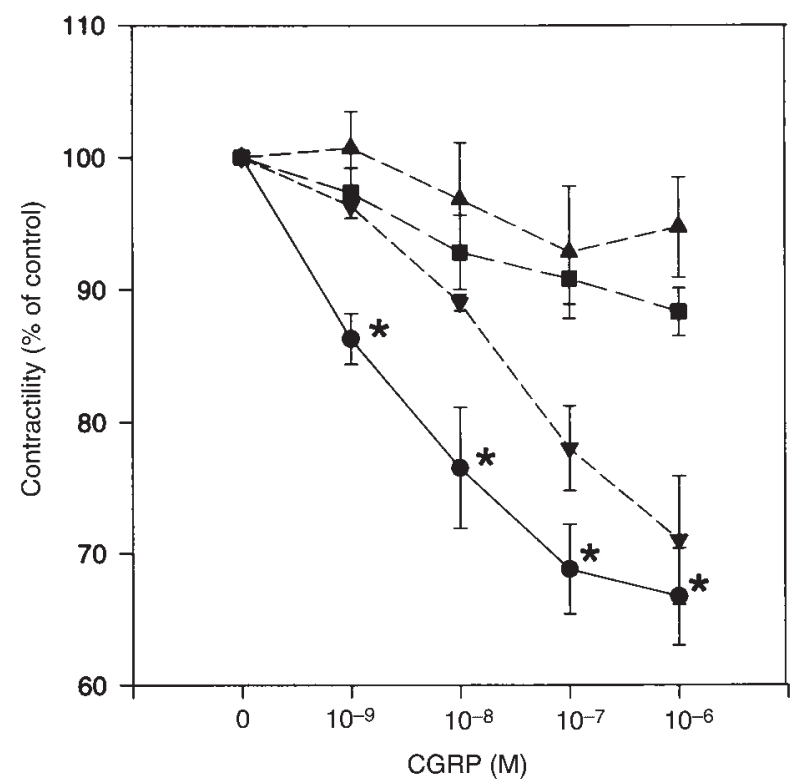

Figure 6

CGRP dose-response curves for relaxation of spontaneously contracting myometrium from pregnant women not in labor. Relaxation responses (expressed as percent of control activity at each dose of CGRP) were analyzed by repeated-measures ANOVA among 4 groups (6 patients each): control (circles), CGRP $_{8-37}$ (squares), $\mathrm{LY}_{83583}$ (triangles), and L-NAME (inverted triangles). Asterisks indicate a statistically significant difference vs. $\mathrm{LY}_{83583}$ and $\mathrm{CGRP}_{8-37}$ groups $(P<0.01$ by ANOVA followed by Bonferroni $t$ test). 


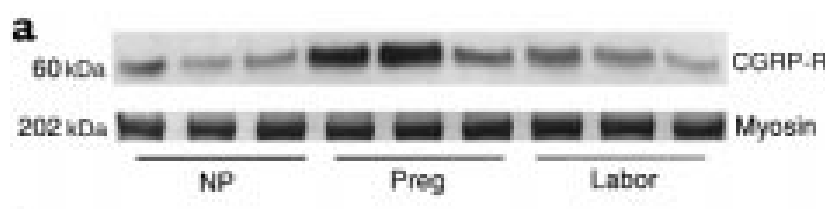

b

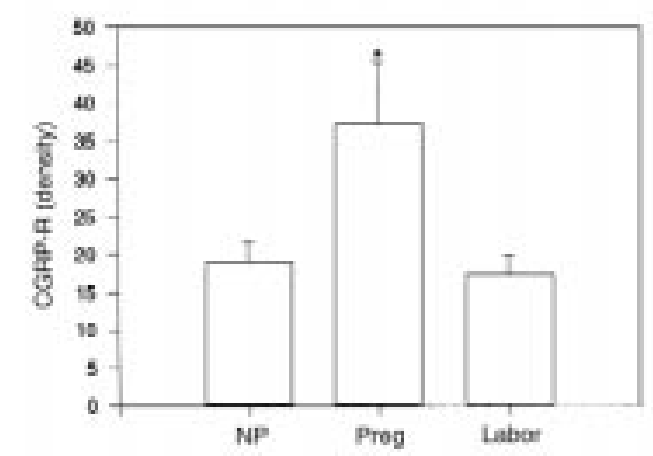

Figure 7

Expression of CGRP-R protein in human myometrium. Top: Western blot of CGRP-R (60 kDa) and myosin $(202 \mathrm{kDa})$ in human myometrium from patients in the 3 treatment groups. Bottom: densitometric measurements of $60-\mathrm{kDa}$ band in Western blots of human myometrium. Each bar represents the mean \pm SEM of 3 patients. Expression in pregnant women (Preg) differs significantly from that in nonpregnant women (NP) and women in labor (Labor) $\left({ }^{*} P<0.01\right.$ by ANOVA).

nant women $(1.55 \pm 0.13)$ compared with nonpregnant women $(0.25 \pm 0.04 ; P<0.01)$, and a precipitous decline during labor $(0.09 \pm 0.03 ; P<0.01)$. These data indicate that the expression of CGRP-R protein and CGRP-R mRNA in human myometrium is minimal in the nonpregnant state, upregulated during pregnancy, and downregulated during term labor.

Localization of CGRP-R in buman myometrium. Using immunofluorescent methods, we found that immunoreactive CGRP-Rs were primarily localized to the myometrial cells in the samples from pregnant women (Figure 9b). Control sections without primary antibody showed no specific staining to these cells in pregnant myometrium (Figure 9e). In contrast to the pregnant uterine sections, the nonpregnant uterine sections showed minimal staining (Figure 9a). Furthermore, both the number of immunopositive cells and the intensity of staining in each cell for CGRP-Rs in the myometrium of women at term labor were reduced compared with samples from pregnant women who were not in labor (Figure 9c). Similarly, control sections without primary antibody showed no specific staining in the segments from nonpregnant women or from pregnant women during labor (Figure 9, d and f).

\section{Discussion}

CGRP has been reported to be involved in the regulation of blood pressure (18) and myocardial contractility (19) through its potent smooth muscle relaxant property, but its action as a mediator of smooth muscle relaxation in human uterus is poorly understood. In this study, we showed that CGRP significantly inhibits both spontaneous and oxytocin-induced contraction of the myometrium during pregnancy. The relaxation effects of CGRP were decreased in myometrium obtained from patients during labor and in the nonpregnant state. CGRP-induced relaxation is inhibited by a CGRP-R antagonist $\left(\mathrm{CGRP}_{8-37}\right)$, a nitric oxide synthase inhibitor (L-NAME), and a soluble guanylate cyclase inhibitor $\left(\mathrm{LY}_{83583}\right)$, indicating the specificity of CGRP effects and possible involvement of the nitric oxide and cGMP pathways. CGRP-Rs are present in human myometrium, and the expression of CGRP-R proteins and mRNA is increased during pregnancy and decreased during term labor. Immunofluorescent staining revealed that CGRP-Rs were abundant in the myometrial cells of pregnant women, and were minimal in the specimens taken from women in labor and in the nonpregnant state. These results indicate that CGRP is involved in the relaxation of human myometrium during pregnancy. Enhanced myometrial sensitivity to CGRP resulting from increased CGRP-R levels in myometrium may play a role in maintaining human myometrium in a quiescent state during pregnancy; a decline in CGRP-Rs at term may facilitate parturition.

CGRP, the most potent endogenously produced smooth muscle relaxant peptide yet discovered, has been found to induce dose-dependent relaxation in spontaneously contracting uterine strips from pregnant rats on day 18 of gestation (9). The relaxation effects of CGRP on the uterus were decreased during spontaneous delivery in the rat at term, compared with effects during preg-

\section{a}

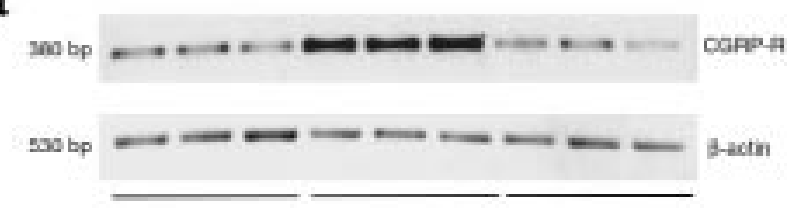

b

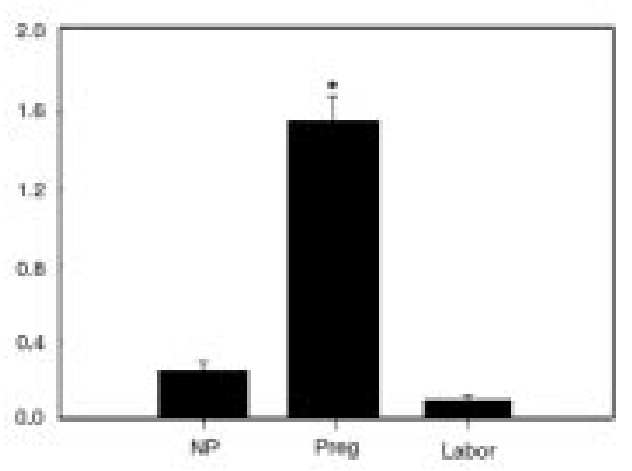

Figure 8

CGRP-R mRNA expression in human myometrium. Top: RT-PCR of samples with CGRP-R primers and $\beta$-actin primers. Bottom: relative levels of CGRP-R mRNA. CGRP-R densities are presented as ratio of densitometric readings of CGRP-R to readings of corresponding $\beta$-actin samples. Each bar represents mean \pm SEM of 3 patients. Expression in pregnant women (Preg) differs significantly from that in nonpregnant women (NP) and women in labor (Labor) $\left({ }^{*} P<0.01\right.$ by ANOVA). 

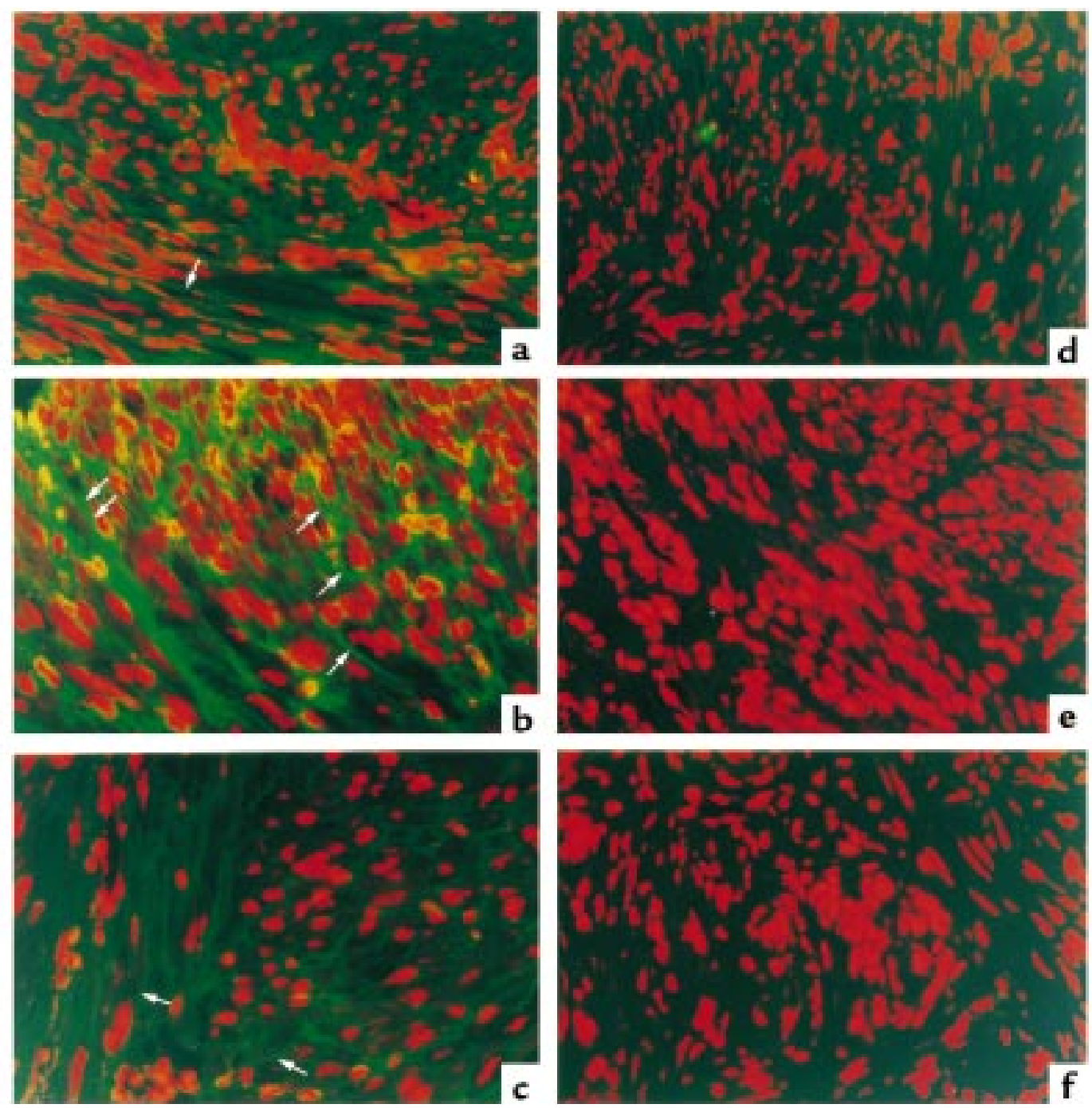

\section{Figure 9}

Immunofluorescent localization of CGRP-R in human myometrium. Left: specimens from nonpregnant women (a), pregnant women not in labor (b), and pregnant women in labor (c). Arrows indicate CGRP-R-positive cells. Right: immunofluorescent staining of human myometrium from negative controls without primary antibody, from nonpregnant women (d), pregnant women not in labor (e), and women in labor (f). Data are representative of results confirmed using a minimum of 3 patients from each group. $\times 400$.

nancy (9). Furthermore, CGRP-containing nerve fibers are present in the human uterus (7), and CGRP relaxes contractile activity in the human uterus and fallopian tubes (7). CGRP is also present in the circulation, and the plasma levels of this neuropeptide are increased during human pregnancy $(5,20)$, indicating that CGRP may play a role in the regulation of human myometrial contractility during pregnancy and labor. We examined the in vitro relaxant effects of CGRP on human myometrium using concentrations of CGRP from $10^{-11}$ to $10^{-6} \mathrm{M}$, based upon the physiologic concentration of circulatory CGRP in humans $\left(10^{-10} \mathrm{M}\right.$; refs. 5 and 20$)$. However, we did not observe significant relaxant effects from CGRP until a concentration of $10^{-9} \mathrm{M}$ was reached. Therefore, all our subsequent experiments were performed using CGRP at a concentration of $10^{-9}$ to $10^{-6} \mathrm{M}$. This study demonstrated increased sensitivity of the myometrium to CGRP; this may contribute to uterine quiescence during pregnancy. Because CGRP also attenuated the oxytocin-stimulated contractions of the myometrium in a dose-dependent manner, this peptide could contribute to uterine quiescence by antagonizing the actions of uterine stimulants such as oxytocin. During labor at term, the sensitivity to CGRP is markedly decreased. Thus, the loss of CGRP-mediated inhibition of uterine contractility at term may allow stimulatory factors to fully activate myometrial contractile activity. The mechanisms for relaxation of human uterine smooth muscle by CGRP remain unclear. It has been suggested that this action occurs through the CGRP-Rs on myometrial smooth muscle cells (21). In the vascular tissues, CGRP induces vasodilation by binding to 2 classes of CGRP-R (22). CGRP-1 receptors are sensitive to the antagonistic action of the $\mathrm{COOH}$-terminal fragment of CGRP (CGRP 8 -37), whereas CGRP-2 receptors are not. Given the significant inhibition of relaxant effects by 
CGRP $_{8-37}$ in this study, it appears that CGRP-1 receptors are present in the human myometrium. The loss of the inhibitory capacity of CGRP on myometrial contractility during labor appears to originate at the receptor-transcription level. This is indicated by the observations that the expression of CGRP-R proteins and CGRP-R mRNA in myometrium is increased during pregnancy and decreased during labor at term. In addition, immunofluorescent staining revealed that CGRP-Rs were abundant in the myometrial cells of pregnant women, and were minimal in the specimens taken from women in labor. A correlation between the levels of CGRP-Rs in human myometrium and the relaxant capacity of CGRP on myometrium further supports the notion that changes in CGRP-R levels are an essential component of the CGRP signaling pathway in the human uterus.

It has been proposed that activation of cGMP and nitric oxide release are involved in the mechanisms of CGRP action in rat uterine relaxation (9). Whether these alternate mechanisms coexist and work synergistically in myometrial relaxation in humans is unknown. Our results show that CGRP-induced relaxation of human myometrium is inhibited by a soluble guanylate cyclase inhibitor $\left(\mathrm{LY}_{83583}\right)$, suggesting that the cGMP pathway may be involved in CGRP-induced relaxation. Exposure of the myometrial strips to an inhibitor of nitric oxide synthase (L-NAME) at a negative log concentration of $3.5 \mathrm{~mol} / \mathrm{L}$ demonstrated a $50 \%$ inhibition of the relaxation effect of CGRP, indicating that nitric oxide may play a role in the postreceptor pathway of CGRP action. Several studies have suggested that the action of CGRP in vasodilation is independent of endothelium $(22,23)$. It appears that CGRP interacts directly with receptors in smooth muscle cells that are coupled to cAMP production $(5,24)$. In the rat aorta, however, CGRP exerts endothelium-dependent relaxant effects by releasing nitric oxide, which stimulates soluble guanylate cyclase to raise cGMP levels in the vascular smooth muscle cells (22). Because the effects of L-NAME on CGRP-induced uterine relaxation were dose-dependent (Figure 6, Table 1 ), we suggest that CGRP action in the human myometrium may involve the nitric oxide pathway. Irrespective of the postreceptor mechanisms, CGRP may be involved in human myometrial relaxation during pregnancy through increased CGRP-R levels.

\section{Acknowledgments}

Financial support from the National Institutes of Health (grants HL-58144 and HD-30273) is gratefully acknowledged.

1. Soloff, M.S., Alexandrova, M., and Fernstrom, M.J. 1979. Oxytocin receptors: triggers for parturition and lactation? Science. 204:1313-1315.
2. Garfield, R.E., and Yallampalli, C. 1993. Control of myometrial contractility and labor. In Basic mechanisms controlling term and preterm birth. K. Chwalisz and R.E. Garfield, editors. Springer-Verlag. Berlin, Germany. $1-28$.

3. Mhaouty, S., et al. 1995. Characteristics of the alpha 2/beta 2-adrenergic receptor-coupled adenylyl cyclase system in rat myometrium during pregnancy. J. Biol. Chem. 270:11012-11016.

4. Yallampalli, C., Dong, Y.L., Gangula, P.R.R., and Fang, L. 1998. Role and regulation of nitric oxide in the uterus during pregnancy and parturition. J. Soc. Gynecol. Investig. 5:58-67.

5. Wimalawansa, S.J. 1996. Calcitonin gene-related peptide and its receptors: molecular genetics, physiology, pathophysiology, and therapeutic potentials. Endocr. Rev. 17:533-585.

6. Mandsager, N.T., Brewer, A.S., and Myatt, L. 1994. Vasodilator effects of parathyroid hormone, parathyroid hormone-related protein, and calcitonin gene-related peptide in the human fetal-placental circulation. $J$. Soc. Gynecol. Investig. 1:19-24.

7. Samuelson, U.E., Dalsgaard, C.J., Lundberg, J.M., and Hokfelt, T. 1985. Calcitonin gene-related peptide inhibits spontaneous contractions in human uterus and fallopian tube. Neurosci. Lett. 62:225-230.

8. Shew, R.L., Papka, R.E., and McNeill, D.L. 1992. Galanin and calcitonin gene-related peptide immunoreactivity in nerves of the rat uterus: localization, colocalization, and effect on uterine contractility. Peptides. 13:273-279.

9. Dong, Y.L., Gangula, P.R.R., Fang, L., Wimalawansa, S.J., and Yallampalli, C. 1998. Uterine relaxation responses to calcitonin gene-related peptide and calcitonin gene-related peptide receptors decreased during labor in rats. Am. J. Obstet. Gynecol. 179:497-506.

10. Dong, Y.L., Gangula, P.R.R., and Yallampalli, C. 1996. Nitric oxide synthase isoforms in the rat uterus: differential regulation during pregnancy and labor. J. Reprod. Fertil. 107:243-254.

11. Wimalawansa, S.J., Gunasekera, R.D., and Zhang, F. 1993. Isolation, purification and characterization of calcitonin gene-related peptide receptor. Peptides. 14:691-699.

12. Wimalawansa, S.J. 1991. Isolation, purification and biochemical characterization of calcitonin gene-related peptide receptor. Ann. NY Acad. Sci. 657:70-87.

13. Morara, S., Wimalawansa, S.J., and Rosina, A. 1998. Monoclonal antibodies reveal expression of the CGRP receptor in Purkinje cells, interneurons and astrocytes of rat cerebellar cortex. Neuroreport. 9:3755-3759.

14. Chomczynski, P., and Sacchi, N. 1987. Single-step method of RNA isolation by acid guanidinium thiocyanate-phenol-chloroform extraction. Anal. Biochem. 162:156-159.

15. Dong, Y.L., Dai, B.S., Singh, P., and Yallampalli, C. 1997. Involvement of nitric oxide pathway in prostaglandin F $2 \alpha$-induced preterm labor in rats. Am. J. Obstet. Gyencol. 177:907-918.

16. Aiyar, N., et al. 1996. A cDNA encoding the calcitonin gene-related peptide type 1 receptor. J. Biol. Chem. 271:11325-11329.

17. Nudel, U., et al. 1983. The nucleotide sequence of the rat cytoplasmic beta-actin gene. Nucleic Acids Res. 11:1759-1771.

18. Nelson, M.T., Huang, Y., Brayden, J.E., Hescheler, J., and Standen, N.B. 1990. Arterial dilations in response to calcitonin gene-related peptide involve activation of $\mathrm{K}+$ channels. Nature. 344:770-773.

19. McCulloch, J., Uddman, R., Kingman, T.A., and Edvinson, L. 1986. Calcitonin gene-related peptide: functional role in cerebrovascular regulation. Proc. Natl. Acad. Sci. USA. 83:5731-5735.

20. Stevenson, J.C., MacDonald, D.W.R., Warren, R.C., Booker, M.W., and Whitehead, M.I. 1986. Increased concentration of circulating calcitonin gene-related peptide during normal human pregnancy. Br. Med. J. 293:1329-1330.

21. Tritthart, H.A. 1992. Membrane actions of calcitonin gene-related peptide in cardiac and smooth muscle myocytes. Ann. NY Acad. Sci. 657:216-227.

22. Zaidi, M., Moonga, B.S., Bevis, P.J., Bascal, Z.A., and Breimer, L.H. 1990. The calcitonin gene peptides: biology and clinical relevance. Crit. Rev. Clin. Lab. Sci. 28:109-174.

23. Nelson, S.H., Steinsland, O.S., and Suresh, M.S. 1993. Possible physiologic role of calcitonin gene-related peptide in the human uterine artery. Am. J. Obstet. Gyencol. 168:605-611.

24. Fiscus, R.R., Wang, X., and Hao, H. 1992. hCGRP . $_{87}$ antagonizes vasodilations and cAMP responses to rat CGRP in rat caudal artery. Ann. NY Acad. Sci. 657:513-515. 